Kacper Topolnicki · Jacek Golak • Roman Skibiński • Alaa Eldeen Elmeshneb · Henryk Witała • Andreas Nogga • Hiroyuki Kamada

\title{
2N and 3N Systems in a Three Dimensional Formalism
}

Received: 5 November 2013 / Accepted: 20 December 2013 / Published online: 7 January 2014

(C) The Author(s) 2014. This article is published with open access at Springerlink.com

\begin{abstract}
We present an overview of our framework used to treat two- and three-nucleon $(2 \mathrm{~N}, 3 \mathrm{~N})$ systems employing three dimensional momentum eigenstates. Using a three dimensional formalism instead of the classical partial wave approach is an attractive alternative for a number of reasons, the most prominent being the very direct way of performing calculations. With the use of our tools it is possible to produce a working numerical realization of calculations in only a couple of steps from the fundamental (Schrödinger or Lippmann-Schwinger) equations. The FORTRAN implementations of the most complicated parts of the calculations are generated automatically by Mathematica ${ }^{\circledR}$ software that was written in our group. Additionally, at higher energies, three dimensional calculations avoid problems arising from slow convergence of partial wave decomposition based techniques. Our approach utilizes a very general form of the $2 \mathrm{~N}$ and $3 \mathrm{~N}$ forces and has been successfully used to obtain results for the $2 \mathrm{~N}$ transition operator as well as for the $2 \mathrm{~N}$ and $3 \mathrm{~N}$ bound states (Golak et al. in Phys Rev C 81:034006, 2010; Few-Body Syst 53:237, 2012a; Few-Body Syst, 2012b).
\end{abstract}

\section{Introduction}

A numerical realization of calculations involving the $2 \mathrm{~N}$ and $3 \mathrm{~N}$ bound state and the transition operator is classically achieved by using partial wave projected operators. We explore a different approach that involves three dimensional momentum vectors. Our calculations are performed using automatically generated FORTRAN codes. These codes contain implementations of the complicated algebraic expressions that result from the momentum dependence of spin matrix elements and can in some cases have the volume of many thousands of lines. The possibility of encapsulating the most complicated parts of the calculation in an automatically generated code allows us to create a numerical realization that is very directly related to the fundamental equations.

Working within the isospin formalism to describe the few (spin $\frac{1}{2}$, isospin $\frac{1}{2}$ ) nucleon systems makes it possible to calculate matrix representations of all relevant momentum projected spin operators using the Kronecker product. Permutations within the spin (isospin) space are explicitly represented as $4 \times 4$ (for $2 \mathrm{~N}$ systems) or $8 \times 8$ (for $3 \mathrm{~N}$ systems) dimensional matrices. These properties, together with momentum space permutations (of relative momentum eigenstates for the $2 \mathrm{~N}$ system or Jacobi momenta eigenstates for the

K. Topolnicki $(\varangle) \cdot$ J. Golak · R. Skibiński · A. E. Elmeshneb · H. Witała

M. Smoluchowski Institute of Physics, Jagiellonian University, Kraków, Poland

E-mail: kacper.topolnicki@uj.edu.pl

A. Nogga

Institut für Kernphysik, Institute for Advanced Simulation and Jülich Center for Hadron Physics,

Forschungszentrum Jülich, Jülich, Germany

H. Kamada

Department of Physics, Faculty of Engineering, Kyushu Institute of Technology, Kitakyushu, Japan 
$3 \mathrm{~N}$ system) are gathered in our custom Mathematica ${ }^{\circledR}$ software. Our symbolic programs also supply definitions that provide methods to create ready-to-use implementations of Mathematica ${ }^{\circledR}$ expressions in the form of FORTRAN modules or functions. The resulting code can be compiled "as is" and linked directly to the main program. Our symbolic software is flexible, allowing us to optimize the code from the level of Mathematica ${ }^{\circledR}$. Additional optimization is performed afterwards by the FORTRAN compiler. Using these tools it is convenient to perform the most complex parts of the calculation.

\section{Formal Ingredients}

In order to arrive at a practical realization of our calculations we utilize a general, parity and time reversal symmetric, form of the $2 \mathrm{~N}$ potential operator:

$$
\left\langle\boldsymbol{p}^{\prime}\left|\left\langle\gamma^{\prime}\left|\check{V}_{2 \mathrm{~N}}^{\gamma}\right| \gamma\right\rangle\right| \boldsymbol{p}\right\rangle=\delta_{\gamma \gamma^{\prime}} \sum_{i=1}^{6} v_{i}^{\gamma}\left(\left|\boldsymbol{p}^{\prime}\right|,|\boldsymbol{p}|, \hat{\boldsymbol{p}}^{\prime} \cdot \hat{\boldsymbol{p}}\right)\left[\check{w}_{i}\left(\boldsymbol{p}^{\prime}, \boldsymbol{p}\right)\right]
$$

where $|\gamma\rangle$ is one of the four possible states $|\gamma\rangle \equiv\left|t m_{t}\right\rangle$ of the total $2 \mathrm{~N}$ isospin, $\check{w}_{i}\left(\boldsymbol{p}^{\prime}, \boldsymbol{p}\right)$ is a set of spin operators (they are listed for example in [7]) and finally, $v_{i}^{\gamma}\left(\left|\boldsymbol{p}^{\prime}\right|,|\boldsymbol{p}|, \hat{\boldsymbol{p}}^{\prime} \cdot \hat{\boldsymbol{p}}\right)$ is a set of scalar functions of the magnitude of the final and initial relative momentum and the angle between them. A very similar decomposition is used for the transition operator $\check{t}$ but with $v_{i}^{\gamma}(\ldots) \rightarrow t_{i}^{\gamma}(E, \ldots)$. The momentum space matrix elements of any $3 \mathrm{~N}$ potential operator can also be written as a finite linear combination of scalar functions of momentum vectors and momentum dependent spin operators, including additionally a limited set of $3 \mathrm{~N}$ isospin operators.

We use a well known operator form of the $2 \mathrm{~N}$ bound state:

$$
\left\langle\boldsymbol{p} \mid \phi_{\mathrm{d}}\right\rangle=\sum_{l=1}^{2} \phi_{l}(|\boldsymbol{p}|)\left[\check{b}_{l}(\boldsymbol{p})\left|1 m_{d}\right\rangle\right]
$$

where $\boldsymbol{p}$ is the relative momentum of the two nucleons, $\breve{b}_{l}(\boldsymbol{p})$ are spin space operators (listed for example in [7]) and finally $\left|1 m_{d}\right\rangle$ is a state in which the spins of the two nucleons are coupled to a total spin 1 with projection $m_{d}$ (the isospin of the deuteron state is 0 ). For the three nucleon bound state we use [12]:

$$
\langle\boldsymbol{p} \boldsymbol{q}|\langle t T \mid \Psi\rangle=\sum_{i=1}^{8} \Phi_{t T}^{(i)}(|\boldsymbol{p}|,|\boldsymbol{q}|, \hat{\boldsymbol{p}} \cdot \hat{\boldsymbol{q}})\left[\check{O}_{i}(\hat{\boldsymbol{p}}, \hat{\boldsymbol{q}})\left|\chi^{m}\right\rangle\right]
$$

where $\boldsymbol{p}, \boldsymbol{q}$ are Jacobi momenta, $\check{O}(\hat{\boldsymbol{p}}, \hat{\boldsymbol{q}})$ are operators in the $3 \mathrm{~N}$ spin space (listed e.g. in [9]), $\langle t T \mid \Psi\rangle$ is the $3 \mathrm{~N}$ bound state projected onto a state with total isospin $T$ and $\left|\chi^{m}\right\rangle$ is a specific spin state (for details see [9]) of the three particles with a projection $m$.

Inserting the above given operator representations into the fundamental (Schrödinger, Lippmann-Schwinger) equations, and removing the isospin-spin dependencies, all calculations turn into linear problems for the scalar functions $t, \phi, \Phi$. Expressions inside square brackets [...] that appear in (1), (2) and (3) have a matrix representation that can be easily created using our Mathematica ${ }^{\circledR}$ tools. The complicated expressions resulting from combining expressions inside [...] can then be transformed into a ready-to-use FORTRAN code. Finally, the numerical realization of calculations is nicely separated from the encapsulated complex expressions.

\section{Numerical Treatment of Large Linear Operators}

The dimension of the resulting linear operators is, in some cases, vary large. This is especially evident for $3 \mathrm{~N}$ systems where, in order to describe the Faddeev component of the bound state with a reasonable accuracy, approximately $10^{6}$ combinations of argument values of $\Phi$ from (3) are necessary. In order to deal with the large dimension of the linear space we use Krylov subspace methods. Given an operator $\check{A}$ and a starting vector $\boldsymbol{v}$, we work with the projections onto the Krylov subspace:

$$
K(\check{A}, \boldsymbol{v})=\operatorname{span}(\boldsymbol{v}, \check{A} \boldsymbol{v}, \check{A} \check{A} \boldsymbol{v}, \ldots) .
$$


Each consecutive vector will have components corresponding to the largest eigenvalues amplified, thus choosing to work within $K(\check{A}, v)$ we use "the most relevant" subspace of $\check{A}$. An additional advantage of this scheme is the possibility to carry out the calculations without an explicit matrix representation of $\check{A}$. The construction of the subspace by direct iterations is not numerically stable; in practice we use another iteration scheme, the Arnoldi algorithm $[1,10]$ (which produces the same space but carries out the orthogonalization in parallel to the iterations).

\section{Results and Summary}

Combining the three dimensional treatment of the $2 \mathrm{~N}$ and $3 \mathrm{~N}$ systems with tools that use symbolic programming within the Mathematic ${ }^{\circledR}$ programming language allowed us to create a very direct numerical realization of calculations that involve the $2 \mathrm{~N}$ transition operator as well as the $2 \mathrm{~N}$ and $3 \mathrm{~N}$ bound states. The calculations utilize a very general form of the $2 \mathrm{~N}$ and $3 \mathrm{~N}$ potentials and can be quickly applied to test new models. Especially at higher energies, where calculations based on partial wave decomposition become very difficult, our three dimensional approach is a viable option.

Table 1 Comparison between the partial wave and three dimensional treatment of the $3 \mathrm{~N}$ bound state from [9]

\begin{tabular}{lcc}
\hline & PWD & $3 \mathrm{D}$ \\
\hline$\lambda$ & 1.0 & 0.99976 \\
$\left\langle E_{\text {kin }}\right\rangle$ & 33.448 & 33.412 \\
$\left\langle E_{\text {pot }}^{2 \mathrm{~N}}\right\rangle$ & -41.329 & -41.273 \\
$\left\langle E_{\text {pot }}^{3 \mathrm{~N}}\right\rangle$ & -0.765 & -0.770 \\
Total energy & -8.646 & -8.631 \\
\hline
\end{tabular}

The energy argument of $\check{K}(E)$ was chosen to be $-8.646(\mathrm{MeV})$. The expectation values of the kinetic and potential energies (given in $\mathrm{MeV}$ ) are in good agreement. The calculations use chiral NNLO $2 \mathrm{~N}$ and $3 \mathrm{~N}$ potentials from [5,6] and are based on np interactions only, therefore the energy differs from the experimental value
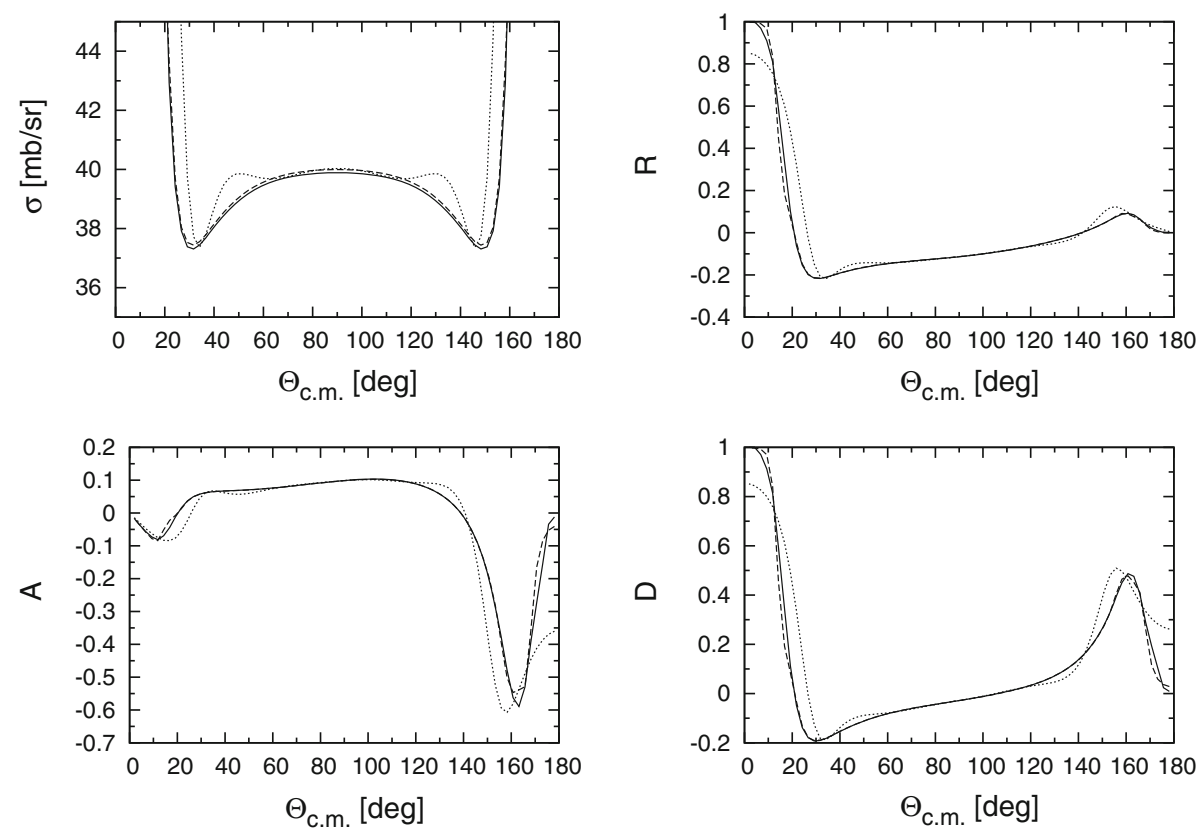

Fig. 1 Selected observables for the proton-proton system (including the Coulomb force) at the projectile laboratory kinetic energy $13 \mathrm{MeV}$ as a function of the center-of-mass angle $\theta_{c . m}$. for the chiral proton-proton NNLO potential $[4,6]$. Results are obtained by solving the Lippmann-Schwinger equation using the matrix inversion method and applying the exponential screening (4) to the Coulomb potential. Dotted, dashed and solid lines show the results with the screening parameter $R=20,60$ and $120 \mathrm{fm}$, respectively, all for $n=4$. Figures are from [8] 
Carrying out matrix-vector type operations that are needed, for example, in the three dimensional treatment of the $3 \mathrm{~N}$ bound state and transition operator, require the use of powerful computing resources. We use the JUQUEEN cluster in FZJ. The results are in good agreement with partial wave calculations. Both of these approaches involve solving a linear eigensystem in the form $\check{K}(E) \phi=\lambda \phi$ and finding a value of the bound state energy $E$ such that $\lambda \approx 1$. Table 1 shows a comparison between the partial wave and three dimensional treatment of the problem, where the energy argument of $\check{K}(E)$ was chosen to be $-8.646(\mathrm{MeV})$. One can see that the results are in agreement with each other.

An example of the three dimensional treatment of proton-proton scattering, taken from [8], is shown in Fig. 1. This example shows that in addition to the short ranged chiral proton-proton NNLO potential $[4,6]$ also the Coulomb interaction can be taken into account using screening. For the case presented in the plots exponential screening was used. The additional contribution to the two nucleon potential is proportional to [8]:

$$
s_{1}(r ; n, R) \equiv \exp \left(-\left(\frac{r}{R}\right)^{n}\right)
$$

where $r$ is the relative position of the two particles, $R$ is the screening parameter and we limit ourselves to the case where $n=4$. The momentum space expressions for the Coulomb potential with (4) can be found in [11]. It can be observed that the results become independent from the screening parameter.

We presented results for the $3 \mathrm{~N}$ bound state and for pp scattering obtained within calculations that are based on a purely $3 \mathrm{D}$ treatment on the problem without PWD. In our formulation, we nevertheless make use of the rotational invariance by means of an expansion in a set of operators. We showed that the obtained high dimensional set of linear equations for the scalar functions accompanying these operators can be reliably solved. These are important steps towards an application in the three-nucleon continuum, which at high energies will benefit from an improved accuracy when the PWD can be circumvented. The new formulation is especially interesting, since the most involved parts of the codes can be generated by Mathematica ${ }^{\circledR}$ scripts. This makes the incorporation of new structures in the interactions much easier by avoiding long developments for partial wave decomposed interactions. This will be important for applying new $3 \mathrm{NF}$ structures as formulated in $[2,3]$.

Acknowledgments We acknowledge support by the Foundation for Polish Science MPD program, conanced by the European Union within the European Regional Development Fund. Part of the numerical calculations have been preformed on the Supercomputing cluster of the JSC, Jülich, Germany. This work was supported by the Polish National Science Center under Grant No. DEC-2011/01/B/ST2/00578. It was also partially supported by the European Community-Research Infrastructure Integrating Activity "Exciting Physics Of Strong Interactions" (acronym WP4 EPOS) under the Seventh Framework Programme of EU.

Open Access This article is distributed under the terms of the Creative Commons Attribution License which permits any use, distribution, and reproduction in any medium, provided the original author(s) and the source are credited.

\section{References}

1. Arnoldi, W.E.: The principle of minimized iterations in the solution of the matrix eigenvalue problem. Q. Appl. Math. 9, $1729(1951)$

2. Bernard, V., Epelbaum, E., Krebs, H., Meißner, U.-G.: Subleading contributions to the chiral three-nucleon force: long-range terms. Phys. Rev. C 77, 064004 (2008)

3. Bernard, V., Epelbaum, E., Krebs, H., Meißner, U.-G: Subleading contributions to the chiral three-nucleon force. II. Shortrange terms and relativistic corrections. Phys. Rev. C 84, 054001 (2011)

4. Epelbaum, E.: Private communication

5. Epelbaum, E., Nogga, A., Glöckle, W., Kamada, H., Meißner, U.-G., Witała, H.: Three-nucleon forces from chiral effective field theory. Phys. Rev. C 66, 064001 (2002)

6. Epelbaum, E., Glöckle, W., Meißner, U.-G.: The two-nucleon system at next-to-next-to-next-to-leading order. Nucl. Phys. A 747, $362(2005)$

7. Golak, J., Glöckle, W., Skibiński, R., Witała, H., Rozpędzik, D., Topolnicki, K., Fachruddin, I., Elster, Ch., Nogga, A.: The two-nucleon system in three dimensions. Phys. Rev. C 81, 034006 (2010)

8. Golak, J., Skibiński, R., Witała, H., Topolnicki, K., Glöckle, W., Nogga, A., Kamada, H.: Different methods for the twonucleon t-matrix in the operator form. Few-Body Syst. 53, 237 (2012a)

9. Golak, J., Topolnicki, K., Skibiński, R., Glöckle, W., Kamada, H., Nogga, A.: A three-dimensional treatment of the threenucleon bound state. Few-Body Syst. (2012b). doi:10.1007/s00601-012-0472-5

10. Saad, Y.: Iterative Methods for Sparse Linear Systems. 2nd edn. SIAM, Philadelphia (2003)

11. Skibiński, R., Golak, J., Witała, H.: Numerical investigations of the three-dimensional proton-proton screened Coulomb t-matrix. Acta Phys. Polon. B 41, 875-895 (2010)

12. Wolfenstein, L.: Possible triple-scattering experiments. Phys. Rev. 96(6), 1654-1658 (1954) 